\title{
On the Quality of Three-Dimensional Electron Diffraction Data for Direct Location of Guest Molecules in Open-Framework Materials
}

\author{
Meng Ge, Zhehao Huang, Hongyi Xu, and Xiaodong Zou* \\ Department of Materials and Environmental Chemistry, Stockholm University, 10691 Stockholm, Sweden \\ gemeng733@gmail.com
}

\begin{abstract}
Can the location of the guest molecules inside the pores of porous materials be determined using three-dimensional electron diffraction (3DED) technique? In most cases, the guest molecules cannot be located from the 3DED data. There are few cases where the positions of guest molecules have been successfully localized by combining 3DED with powder X-ray diffraction (PXRD). Simulated annealing (global optimization) and Rietveld refinement are always applied during the refinement procedure. By applying the advanced 3DED method, namely continuous rotation electron diffraction (cRED), it is possible to refine against 3DED data to visualize the conformation of the molecular backbone of guest molecules in a difference Fourier map. However, the individual atomic positions of the guest molecules have not been identified accurately yet. Here, we demonstrate for the first time that by applying cRED, each non-Hydrogen atom from the guest molecules can be separately localized from the difference Fourier map. We choose two open framework germanates, SU-8 and SU-68, as examples. Low electron dose was combined with ultrafast cRED data collection to minimize electron beam damage of the sample. As a result, atoms from the guest molecules appear as distinct, well-separated peaks in the difference Fourier maps. We also demonstrate that the atomic structure of both the framework and the guest molecules obtained by cRED is as reliable and accurate as that obtained by single-crystal X-ray diffraction (SCXRD).
\end{abstract}

Keywords: Three-dimensional electron diffraction, guest molecules, open-framework materials 